\title{
Cotside EEG monitoring using computerised spectral analysis
}

\author{
S S AZIZ, S J WALLACE, J F MURPHY, C P Q SAINSBURY, AND O P GRAY \\ Department of Paediatrics, University Hospital of Wales and Department of Child Health, University of \\ Wales College of Medicine, Cardiff
}

SUMMARY The application and interpretation of computerised spectral analysis of the neonatal electroencephalogram (EEG) using the Neuroscience Berg Fourier Analyser (BFA) is described. Recordings are immediately available at the cotside. Electrophysiological changes can be recognised by individuals with no previous experience in EEG technology. The compact nature of the analysis allows long periods of recording to be viewed within minutes. In addition to the unequivocal demonstration of both clinical and subclinical seizures, the BFA is useful in the evaluation of interseizure activity - that is, disturbance of sleep patterns, electrical output, and hemisphere asymmetry.

The newborn who convulses has a poor neurodevelopmental outlook. ${ }^{1}$ Seizures are detected clinically in between 1.4 and 14 per 1000 neonates. ${ }^{1-6}$ Even in the absence of clinical manifestations, however, continuous electroencephalographic (EEG) monitoring has shown that seizure activity is common in ill and particularly preterm newborns. ${ }^{7}$ As such activity may cause neuronal death, ${ }^{8}$ the presence of even subclinical fits is a cause for concern. Conventional EEG recording in the neonate is difficult both technically and in interpretation. Prolonged EEG monitoring with storage on tape and later replay only allows seizures to be detected retrospectively and is unsuitable for the simultaneous evaluation of therapeutic interventions. A pressing need exists for technology that will enable the neonatologist both to detect subclinical seizures as they occur and to monitor responses to treatment. More information is also needed on electrical activity of the brain between seizures.

The present study investigates the practicality and reliability of using compressed spectral analysis of the EEG recorded continuously at the cotside in the management of neonatal seizures.

\section{Materials and methods}

Neuroscience Berg Fourier Analyser (BFA). The BFA is a computerised system that analyses the raw EEG using Berg and Fourier transformations. Both of these computerised mathematical calculations plot the electrical output (amplitude in millivolts), or power, against frequency $\left(\mu \mathrm{V}^{2} / \mathrm{Hz}\right)$. Fourier transformation is the simpler of the two. In this transformation eight second epochs of EEG are analysed, the total power at each frequency being recorded (Fig. 1 (a)). Fourier transformation is particularly suitable for evaluating sleep states, symmetry, and frequencies. Its drawback is that it gives a disproportionate importance to low frequencies, which tend to be of high amplitude. Thus it is not always suitable for detecting seizures as these are often associated with high frequency discharges. Berg transformation (Fig. 1 (b)), on the other hand, is more suitable for detecting seizure discharges in which high frequency changes occur. With this transformation, the epoch is related to the frequency - that is, low frequencies have a long epoch while high frequencies have shorter epochs.

Technique. In order to validate the findings using the BFA, simultaneous EEGs were obtained on tape using a Medilog 24 hour ambulatory EEG monitor (Oxford Instruments). Silver/silver chloride electrodes were applied to the scalp using collodion. On completion of the recording they were removed with acetone/olive oil. Double ended leads were used, one limb being attached to the BFA, the other to the Medilog recorder. Skin/electrode impedance was measured using an SLE recorder (EIT), the value being kept under $5 \mathrm{Kohm}$. The data output of the BFA was recorded on paper running at $1 \mathrm{~mm}$ 


\section{The Fourier transformation}
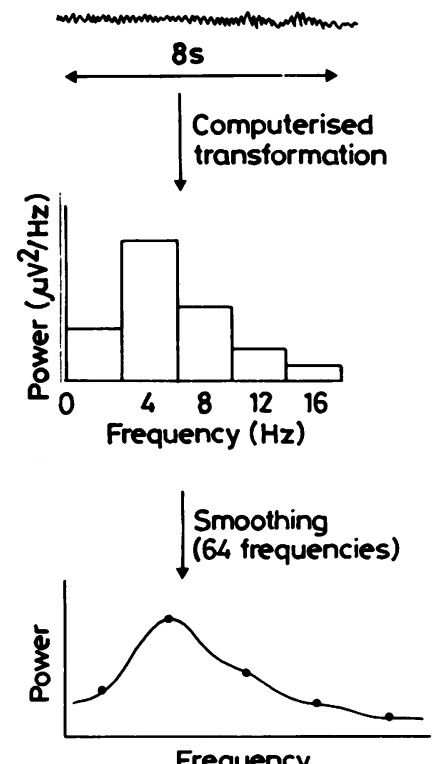

Frequency

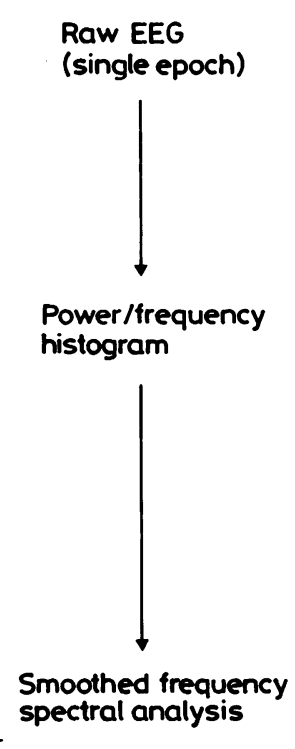

Fig. 1 (a) Fourier transformation of the conventional EEG.

steps. The simultaneously obtained conventional EEG was replayed after completion of recording on a visual display unit (PMD 12 Virgo special, Oxford Medical Systems). All clinical events were noted by one of us (SA) or by attendant trained nursing staff.

\section{Patients.}

\section{Normal infants}

Recordings were made of 10 normal full term infants using the BFA and the Medilog recorder simultaneously. Informed consent was obtained from the mothers. Each baby was studied for a two hour period between feeds. The sleep state was continuously observed clinically by one of us (SA).

\section{Neurologically disturbed infants}

Recordings were also made from sick infants with a variety of problems, including seizures, asphyxia, periventricular haemorrhage, hypoglycaemia, and hydranencephaly.

\section{Results}

The performance of the BFA has been validated by comparing its tracings with simultaneously obtained recordings using the Medilog tape. Figure 2 shows

\section{The Berg transformation}

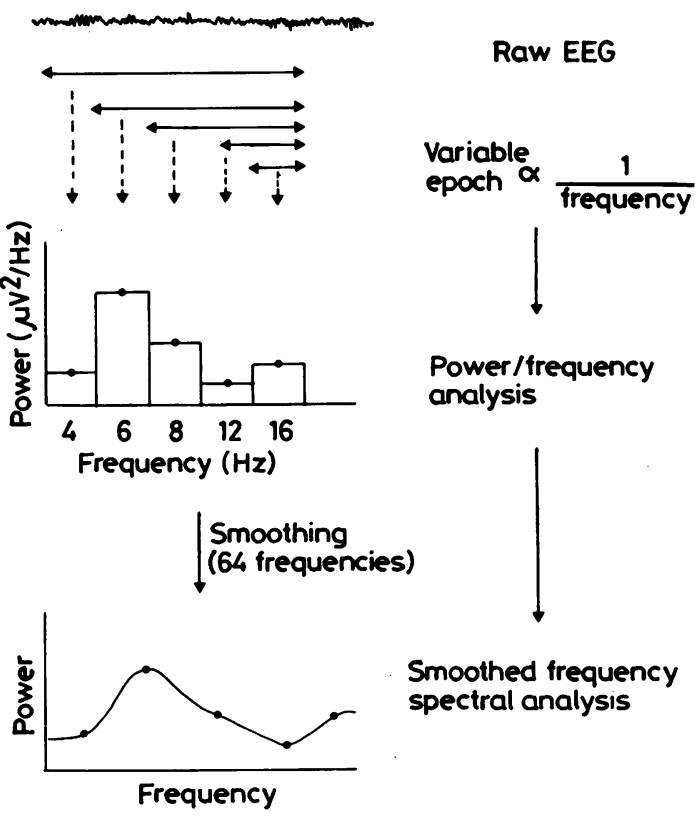

Fig. 1 (b) Berg transformation of the conventional EEG.

examples of the BFA and conventional (Medilog recorded) EEG traces for the same 16 second epoch. In the BFA recording made using the Fourier transformation maximum power is seen in the $2-6 \mathrm{~Hz}$ range in both right and left hemispheres. The conventional EEG likewise shows the highest voltage to be in the slower frequencies. Both the BFA and conventional EEG show much lower voltage (or power) in the higher frequencies. Thus the frequency distribution and amplitude of the EEG are as clearly recognisable on the BFA as on a conventional recording.

Normal term infants. The records obtained from the 10 normal term infants are entirely comparable with one another. Patterns characteristic of quiet and active sleep are readily recognisable. Figure 3 shows a typical recording. On entering a phase of quiet sleep there is a moderately abrupt increase in the power in the $0-16 \mathrm{~Hz}$ range, most pronounced in the lower frequencies, though increased activity up to $10-12 \mathrm{~Hz}$ is also evident. When the infant is in active sleep, although there is a tendency for the power to be slightly greater in the lower frequencies, activities are generally of low power in all frequencies from $0-16 \mathrm{~Hz}$. 
Fig. 2 BFA and conventional EEG recordings (left and right hemispheres) during the same 16 second epoch from a normal full term neonate in quiet sleep.

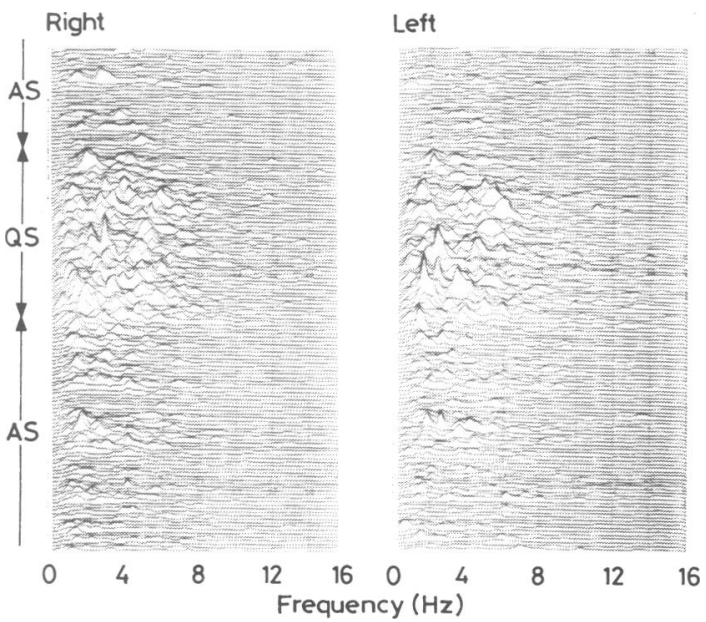

Fig. 3 Compressed spectral analysis (left and right hemispheres) of sequential 16 second epochs of EEG recorded over one hour in a normal full term neonate.

AS $=$ active sleep. $\mathbf{Q S}=$ quiet sleep
Infants with seizures. Seizure discharges are shown in Figure 4. On analysing EEG frequencies between 0 and $16 \mathrm{~Hz}$ normal sleep cycles are not recognisable and bursts of high power, in the $0-8 \mathrm{~Hz}$ frequency range, are visible on one occasion on the right and five occasions on the left side. These bursts coincided with clinical seizures. If the EEG frequencies between 0 and $32 \mathrm{~Hz}$ are analysed subclinical seizure activity, confirmed on the Medilog recorder, can be identified as episodic increases in power in the 16-28 $\mathrm{Hz}$ range. With the exception of the identification of isolated spike discharges seizure activity was as $\mathcal{O}$ readily recognisable with the BFA as with conven- $N$ tional EEG recording. In premature infants the normal slow and fast wave complexes contain a $\omega$ mixture of many frequencies of variable power, whereas seizure discharges tend to be stereotyped and stand out as fairly high power bursts in a restricted number of frequencies. Where seizures are associated with paroxysmal decreases in amplitude of the conventional EEG reduced power is evident on the BFA, but it is difficult to distinguish 

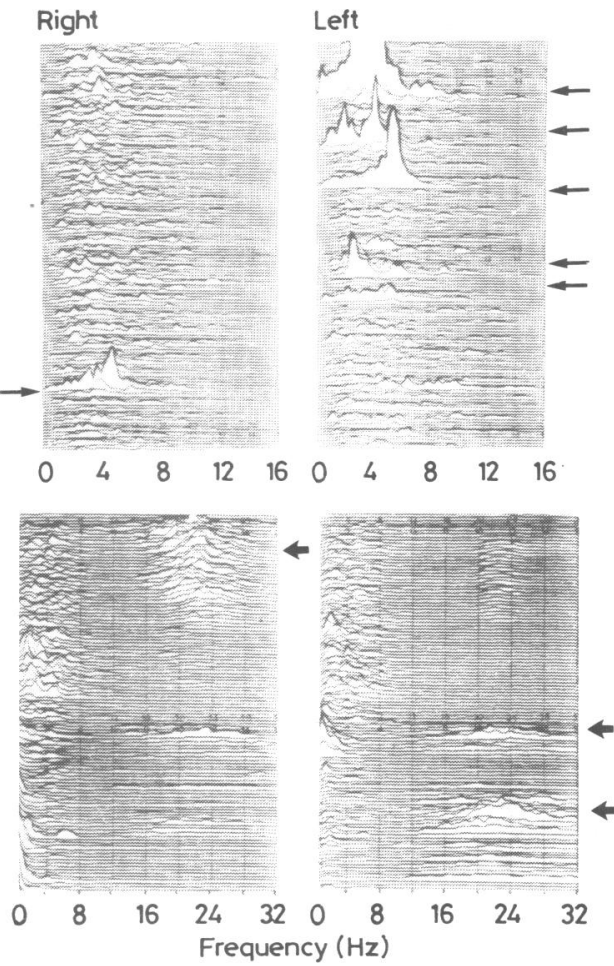

Fig. 4 Compressed spectral analysis (left and right hemispheres) of 16 second epochs of EEG in a full term neonate.

$0-16 \mathrm{~Hz}$ recording: clinical seizures as indicated: 0-32 $\mathrm{Hz}$ subclinical seizures as indicated.

these changes from those of suppression due to any cause.

Identification of artefacts. The BFA automatically indicates artefacts by a small line alongside the relevant epoch.

\section{Discussion}

The particular characteristics of the neonatal EEG and its relations to gestation and to sleep and waking states have been intensively researched. ${ }^{9}$ The changes associated with seizures are also well documented. ${ }^{10}$ Analysis of conventional continuous recordings either on paper or on tape, with subsequent viewing via a display unit, is, however, time consuming. The Neuroscience BFA, which is designed to provide on the spot computerised spectral analysis of the EEG in a compressed form, is a great advance for the practising clinician. The spectral display makes it possible to readily detect seizures and more subtle electrophysiological changes. Once the principles involved in this type of presentation are understood, interpretation of the recordings is fairly easy even for those not aware of the EEG technology.

After analysis, the BFA presents the frequency $(\mathrm{Hz})$ distribution both graphically and numerically. The compact nature of the analysis means that long periods of recording - that is, 24-48 hours - can be viewed in a matter of minutes. The machine notes if artefacts occur and differentiates between body movements and genuine electrophysiological events. The normal neonatal sleep patterns and alterations related to seizures and other pathological states are clearly recognisable. In the normal neonate the EEG patterns characteristic of active and quiet sleep can be seen to merge gradually from one to the other. After a neurological insult-for example, asphyxia-loss of normal sleep patterns is among the earliest of the changes noted and is the last to revert to normal. Clinical seizures are easily confirmed by high power in the low frequencies and subclinical ones are detected in the high frequency range $(20-32 \mathrm{~Hz})$. In the premature infant seizure discharges are distinguishable from normal bursts of activity by their stereotyped nature.

The Neuroscience BFA requires roughly half the area of floor space as that occupied by an incubator and can be accommodated in the neonatal nursery without difficulty. It is mobile but not portable.

In this descriptive study the potential scope of compressed spectral analysis of the neonatal EEG has been shown. The contention that this is a useful additional diagnostic tool in neonatal neurology is supported. In addition, it promises to be a useful means by which intervention studies can be monitored.

We thank Miss J T Gibbs for continuous technical advice and help, the nursing staff of the neonatal wards, and particularly the Special Care Baby Unit, University Hospital of Wales, and the WRVS for donating the Neuroscience Berg Fourier Analyser.

\section{References}

1 Burke JB. The prognostic significance of neonatal convulsions. Arch Dis Child 1954;29:342-5.

2 Eriksson M, Zetterstrom R. Neonatal convulsions: incidence and causes in the Stockholm area. Acta Paediatr Scand 1979;68:807-11.

${ }^{3}$ Craig WS. Convulsive movements occurring in the first 10 days of life. Arch Dis Child 1960:35:226-44.

${ }^{4}$ Keen JH, Lee D. Sequelae of neonatal convulsions. Arch Dis Child 1973:48:542-6.

5 Dennis J. Neonatal convulsions: aetiology, late neonatal status and long term outcome. Dev Med Child Neurol 1978;20:143-58.

${ }^{6}$ Brown JK, Cockburn F, Forfar JO. Clinical and chemical correlates in convulsions of the newborn. Lancet 1972;i:135-9. 
7 Eyre JA, Oozeer RC, Wilkinson AR. Diagnosis of neonatal seizure by continuous recording and rapid analysis of the electroencephalogram. Arch Dis Child 1983;58:785-90.

$\checkmark$ Schwarcz R, Meldrum B. Excitatory amino antagonists provide a therapeutic approach to neurological disorders. Lancet 1985;ii: $140-5$.

${ }^{9}$ Dreyfus-Brissac C. Neonatal electroencephalography. In: Scarpelli EM, Cosmi EV, eds. Reviews in perinatal medicine. Vol 3. New York: Raven Press, 1979:397-466.
${ }^{10}$ Harris R, Tizard JPM. The electroencephalogram in neonatal convulsions. J Pediatr 1960;57:501-20.

Correspondence to Dr S J Wallace, Department of Paediatrics University Hospital of Wales, Heath Park, Cardiff CF4 4XW.

Received 21 October 1985

\section{Fifty years ago}

\section{Parental loss and child guidance}

\section{Muriel Barton Hall and Frederick Hopkins (Liverpool)_Arch Dis Child 1936;11:187-194}

'The personality development of a child depends to a considerable extent upon the influence of its father and its mother.' The total number of children studied was $484 ; 266$ were living with both parents ( 3 boys to 2 girls), 218 with one or none 8 (sexes equal). The majority $(76 \%)$ were between 5 and 14 years old. Behaviour and nervous disorders accounted for $\frac{2}{D}$ four-fifths of the problems for which the clinic was consulted. Behaviour problems predominated among children from $\varrho$ disturbed homes, but intellectual capacity was approximately equal among children from normal and disturbed homes. Behaviour disorders were most pronounced among illegitimate children $(61 \%)$ and less when one or both parents were 3 lost $(53 \%)$ or were absent from home intermittently $(48 \%)$. The equal number of boys and girls from disturbed homes may be due to lack of security in the environment, which affects children regardless of their sex.

'The fact that bchaviour disorders are more prevalent from disturbed homes is considered to be attributable firstly to $\stackrel{\overrightarrow{\mathrm{D}}}{\mathrm{.}}$ the fact of faulty handling through the absence of parents or the presence of substitute parents and, secondly, to the fact that in many cases comparatively mild forms of behaviour disturbance which would be understood by the child's own 0 parents are less readily tolerated by the substitute parents. The uniformity of intellectual development among all the 3 . children suggests that the environmental aspects of the disturbed home are of more significance than the inherent factors. It is concluded that the child from the disturbed home differs as to personality in no ascertainable way from the child from the normal home but there are circumstances in the environment which may lead to symptoms.'

(Dr Hall and Dr Hopkins worked in the Liverpool and District Child Guidance Clinic, and if environmental factors were $D$ of more importance than hereditary ones 50 years ago, even if it was at a time of industrial depression, they are probably even more pronounced in Liverpool today. There is a need for more long term studies comparing children from one $\vec{N}$ parent families, which are now so common, with those from two parent families. Also, to identify risk factors, variations $\Omega$ within one parent families may be important-for example, if they are the result of divorce or of illegitimacy. Nisı. $N$ (GORDON.) 